ARTICLE

https://doi.org/10.1038/s41467-019-12952-6

\title{
Lithiophilic montmorillonite serves as lithium ion reservoir to facilitate uniform lithium deposition
}

\author{
Wei Chen (1) ${ }^{1,2}$, Yin Hu', Weiqiang Lv (1) ${ }^{3}$, Tianyu Lei ${ }^{1}$, Xianfu Wang ${ }^{1}$, Zhenghan Li ${ }^{1}$, Miao Zhang ${ }^{1}$, \\ Jianwen Huang ${ }^{1}$, Xinchuan Du${ }^{1}$, Yichao Yan ${ }^{1}$, Weidong He ${ }^{3,4}$, Chen Liu ${ }^{5}$, Min Liao ${ }^{5 \star}$, Wanli Zhang ${ }^{1}$, \\ Jie Xiong (i) ${ }^{1 \star} \&$ Chenglin Yan (i) ${ }^{2 \star}$
}

The growing demand for lithium batteries with higher energy densities requires new electrode chemistries. Lithium metal is a promising candidate as the anode material due to its high theoretical specific capacity, negative electrochemical potential and favorable density. However, during cycling, low and uneven lithium ion concentration on the surface of anode usually results in uncontrolled dendrite growth, especially at high current densities. Here we tackle this issue by using lithiophilic montmorillonite as an additive in the ether-based electrolyte to regulate the lithium ion concentration on the anode surface and thus facilitate the uniform lithium deposition. The lithiophilic montmorillonite demonstrates a pumping feature that improves the self-concentrating kinetics of the lithium ion and thus accelerates the lithium ion transfer at the deposition/electrolyte interface. The signal intensity of TFSIshows negligible changes via in situ Raman tracking of the ion flux at the electrochemical interface, indicating homogeneous ion distribution, which can lead to a stable and uniform lithium deposition on the anode surface. Our study indicates that the interfacial engineering induced by the lithiophilic montmorillonite could be a promising strategy to optimize the lithium deposition for next-generation lithium metal batteries.

\footnotetext{
${ }^{1}$ State Key Laboratory of Electronic Thin Films and Integrated Devices, University of Electronic Science and Technology of China, Chengdu 610054, China.

${ }^{2}$ College of Energy, Jiangsu Provincial Key Laboratory for Advanced Carbon Materials and Wearable Energy Technologies, Soochow University, Suzhou 215006, China. ${ }^{3}$ School of Physics, University of Electronic Science and Technology of China, Chengdu 610054, China. ${ }^{4}$ National Key Laboratory of Science and Technology on Advanced Composites in Special Environments Center for Composite Materials and Structures Harbin Institute of Technology, Harbin 150080, China. ${ }^{5}$ Hunan Provincial Key Laboratory of Thin Film Materials and Devices, School of Materials Science and Engineering, Xiangtan University, Xiangtan 411105, China. *email: mliao@xtu.edu.cn; jiexiong@uestc.edu.cn; c.yan@suda.edu.cn
} 
ithium metal is the ultimate anode of choice and could push lithium ion batteries to the next performance level ${ }^{1-4}$. Once paired with the high-capacity cathode material (e.g., sulfur ${ }^{5}$, oxygen $^{6}$, etc.), the lithium metal batteries (LMBs) deliver higher specific energy $\left(>500 \mathrm{Wh} \mathrm{kg}^{-1}\right)$ at the cell level and a lower cost (below US $\$ 100 \mathrm{kWh}^{-1}$ ) than the traditional lithium ion batteries $^{7,8}$. The practical application, however, is hindered by $\mathrm{Li}$ plating on the surface of the current collector (e.g., $\mathrm{Cu}$ ) since liquid electrolyte is generally adopted, where the concentration of the $\mathrm{Li}$ ions would drop to zero based on Chazalviel space charge model during cycling 9,10 . Particularly, at high rates, large space charge and electric field will be induced in the vicinity of electrode surface, rendering uneven $\mathrm{Li}$ protuberances ${ }^{11,12}$. During the subsequent deposition process, the protuberances are amplified until dendrites are produced ${ }^{13}$. Thus the practical applications of the LMBs will be blocked by the formed dendrite due to the issues including: (1) the risk of piercing the separator until electronically connecting the electrodes, causing the "soft short"14; (2) the consumption of electrolyte ${ }^{15,16}$; and (3) the decay of battery capacity $^{17,18}$.

Optimizing the structure of current collectors is a typical way to suppress dendritic spread via concealing Li deposits (or dendrites) within the inner of current collector ${ }^{19-21}$. Although those methods show certain degree of controllability in decreasing the risk of short circuit, the dendrite problem appears once the height of Li plating exceeds the thickness of designed current structures. Electrostatic shielding is the other compensating mechanism to reduce the $\mathrm{Li}$ dendritic growth via accommodating the charge distribution ${ }^{22,23}$. Typically, cationic metal salt with special properties is added in the electrolyte to reduce the electric-field intensity on the surface of the current collector caused by uneven three-dimensional (3D) protrusions ${ }^{13,24}$. However, those cations may be seriously reduced due to the low negative electrochemical potential of $\mathrm{Li}$ anode. The reduced cation could be involved in the formation of solid electrolyte interphase (SEI), causing the abatement of electrostatic shielding effect. Beside the above strategies, artificial interface modifications (or solid electrolytes) is another approach to ensure the uniform transport of $\mathrm{Li}$ ions through the artificial layer ${ }^{25-27}$, resulting in uniform nucleation on the surface of the current collector. However, the artificial interface modifications are challenging as a result of the complicated preparation process and harsh conditions, leading to somewhat divorcing from industrial applications. Moreover, the material selection for coating on Li anode needs to be carefully designed with insulating nature and higher shear modulus at least twice than that of the metallic $\mathrm{Li}^{3,28}$. To this end, effective and feasible pathways to suppress $\mathrm{Li}$ dendrites are still urgently required to achieve safe $\mathrm{Li}$ anode for practical application.

Concerning liquid electrolyte (e.g., ether-based electrolyte), the inhomogeneous ion distribution is an important cause of the uneven $\mathrm{Li}$ deposition and thus the generation of $\mathrm{Li}$ dendrite in liquid electrolyte ${ }^{10}$. As shown in Fig. 1a, the lithium ions in the electrolyte deposit onto the surface of the copper current collector during electroplating. However, due to the influence of the lithium ion migration rate in the electrolyte, ions near the electrochemical interface form a double layer ${ }^{29}$. After reduction on the surface of the electrode, the Li ions in the electrolyte cannot replenish the ions at the interface immediately, resulting in the formation of the low concentration regions (Fig. 1a) ${ }^{30}$. Subsequently, Li will selectively deposit in these regions, causing the uncontrollable lithium accumulation. On the contrary, uniform ion distribution (Fig. 1b) can effectively avoid this situation, leading to uniform deposition of the $\mathrm{Li}$ ions based on diffusion model that the electrodeposits display a smooth morphology if ionic concentration profile at the anode evolves to a steady state and high concentration ${ }^{9,10}$.
Montmorillonite, as a member of phyllosilicate, is a classical inorganic material for eliminating heavy metal ion pollution due to the ion self-concentrate that induced higher ionic concentration on its surface rather than that in the bulk solution ${ }^{31,32}$, benefited from its large specific surface area, high ion exchange capability and good adsorption performance. Inspirited by this, herein, by introducing the montmorillonite into the ether-based electrolyte, the lithium ion distribution in the electrolyte can be optimized because of the ionic self-concentrate on the montmorillonite (Fig. 1d), which enable stable and uniform $\mathrm{Li}$ deposition on the $\mathrm{Cu}$ surface, yielding dendrite-free $\mathrm{Li}$ deposits. Compared with the artificial interface modification, the proposed ionic self-concentrating electrolyte is easy to operate, compatible with the mass production, and has the potential to combine with other devices by just replacing the common ether-based electrolyte with the proposed electrolyte without embellishing the lithium anode. Systematic in situ Raman/finite element simulation/other studies reveal the deposition mechanism, providing guidance on the optimization of $\mathrm{Li}$ deposition for nextgeneration LMBs.

\section{Results}

Design of the ion self-concentration electrolyte. The montmorillonite material is single phase according to X-ray powder diffraction (XRD) analysis. The characteristic diffraction peak at $6.12^{\circ}$ (Supplementary Fig. 1) corresponds to an interlayer distance of $1.4 \mathrm{~nm}$, which provides sufficient space within montmorillonite for Li-ion diffusion and electrolyte wetting ${ }^{25}$. After addition of montmorillonite particles, the ether-based electrolyte gradually changes from gray to transparent, implying that the particles cannot be uniformly dispersed in the electrolyte. To ensure uniform distribution of montmorillonite particles in the electrolyte, ionic conducting polymer polyethylene oxide (PEO) was introduced as the framework due to the high binding energy between montmorillonite and PEO (Supplementary Fig. 2). Hereafter, this electrolyte with featured montmorillonite in PEO is referred to as MIP.

Figure 1c, d illustrates the designing concept of tuning the distribution of $\mathrm{Li}$ ions. The adsorption and self-concentrating ability of Li ions on the surface of montmorillonite particles are confirmed with the zeta potential method (Supplementary Fig. 3) via which the adsorbing ion-induced surface potential can be detected. The results show that the montmorillonite possesses a strong affinity for $\mathrm{Li}$ ions with a high zeta potential $(+26 \mathrm{mV}$, the tested electrolyte is based on $20 \mathrm{mM}$ lithium bis(trifluoromethanesulfonyl)imide (LiTFSI) in 1, 3-dioxolane (DOL)) which can adjust the Li distribution through self-concentrate on montmorillonite surface 2,33 , as shown in Fig. 1d. The ion redistribution profile generates a favorable initial interfacial concentration, $\mathrm{C}_{0}$, compared to the pure ether electrolyte (Fig. 1c), effectively delaying Sand's time to suppress initial uneven $\mathrm{Li}$ nucleation under electric field, which is beneficial for forming a stable $\mathrm{Li}$ nucleation. Moreover, no deposit is observed in the bottom of the bottle even after resting for ten days, indicating the dispersion stability of the montmorillonite in the electrolyte (Fig. 1e and Supplementary Fig. 4). The structure stability of the montmorillonite was also confirmed by XRD after being soaked it in electrolyte for $24 \mathrm{~h}$ (Supplementary Fig. 5).

Morphology and evolution of $\mathrm{Li}$ deposition on $\mathrm{Cu}$ negative surface. The plating morphologies of $\mathrm{Li}$ deposition using ether electrolytes with and without MIP are captured to investigate the deposition behavior of Li ions (Fig. 2a-h). The surface of Cu foil was first washed with de-ionized water and ethanol to remove the possible impurities. Before $\mathrm{Li}$ platting, the $\mathrm{Cu}$ surface remains 
a

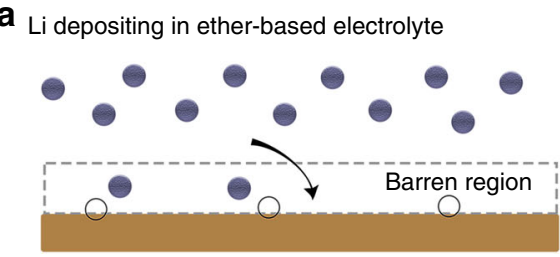

Uneven Li depostion

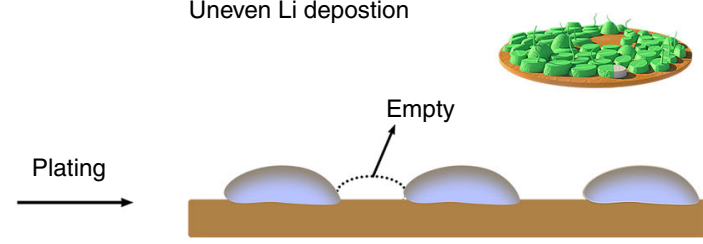

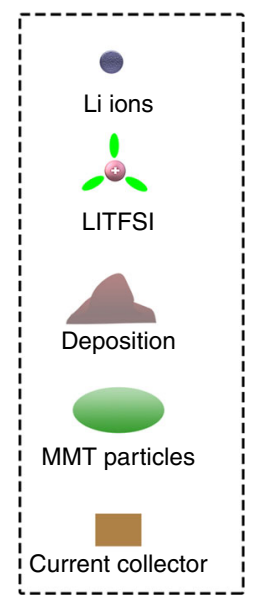

b Li depositing in MIP-based electrolyte

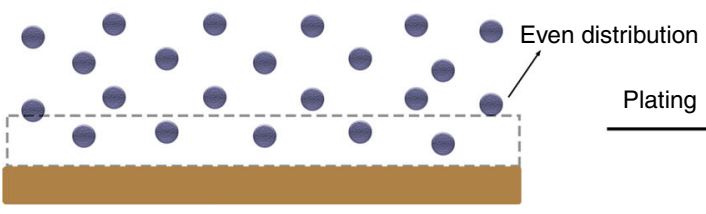

Idealized distribution
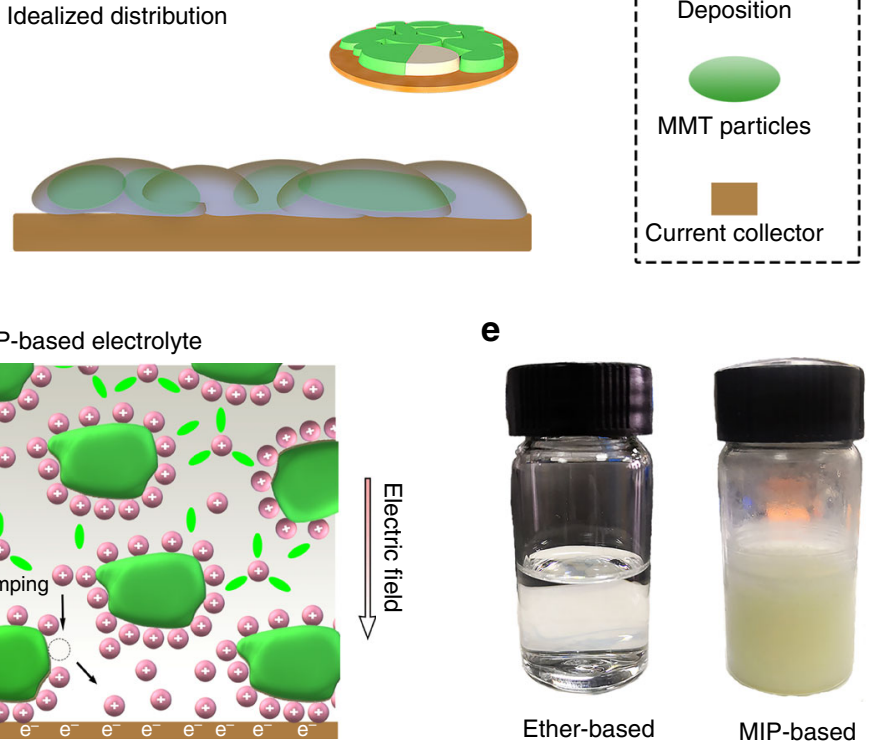

d

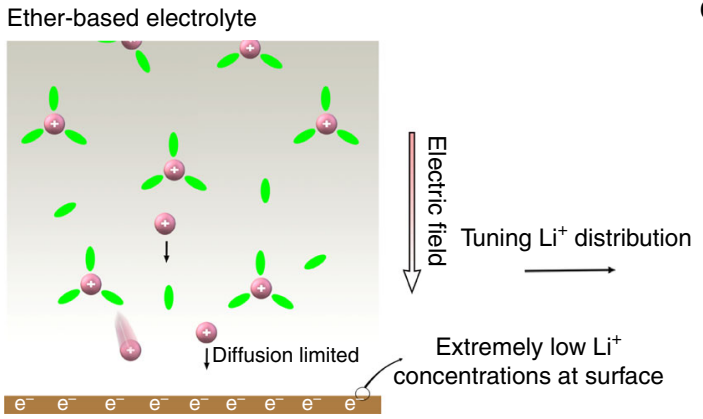

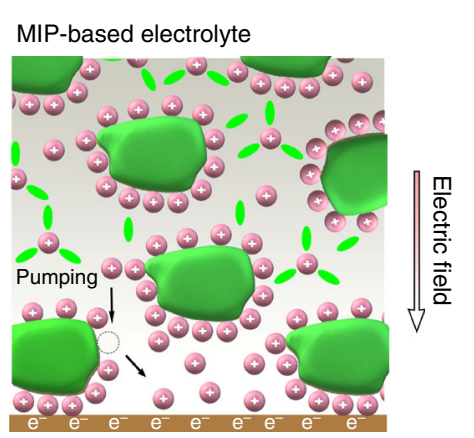

e

Ether-based

MIP-based

Fig. 1 Designing concept of tuning redistribution of Li ion concentration. The nucleation model with barren and rich Li ion concentration at negative surface. Barren $\mathrm{Li}$ ion profile in a makes the nucleation sites uncontrollable, resulting in uneven Li deposits. Rich Li ion profile distribution in $\mathbf{b}$ realize idealized nucleation sites. $\mathbf{c}$ and $\mathbf{d}$ Schematic illustration describing Li ion plating behavior in ether-based and MIP-based electrolyte, respectively. The diffusion limited of $\mathrm{Li}$ ions in ether-based electrolyte results extremely low $\mathrm{Li}$ ion concentrations at $\mathrm{Cu}$ surface under an electric field. Electro-kinetic pumping phenomena in MIP-based electrolyte allows Li ion self-concentrate at the interface of electrode/electrolyte. The arrows in $\mathbf{c}, \mathbf{d}$ represent the movement of the cations. e Optical images of ether- and MIP-based electrolyte

rough and uneven (Supplementary Fig. 6). As shown in Fig. 2a (the inset image), rough surface with a high density of cracks observed in ether-based electrolyte after Li plating with a capacity of $2 \mathrm{mAh} \mathrm{cm}^{-2}$ on $\mathrm{Cu}$ foil. Furthermore, we select two representative regions at the inner (marked red a) and edge (marked red b) of Li deposits to observe the morphology after Li deposition. For the ether-based electrolyte, porous structure with irregular massif-like 3D Li dendrites can be clearly found (Fig. 2b). Even worse, the Li deposits show sharper corners and present seaweed morphology at the edge region (Fig. 2c). It is easier for residual on separator of such sharp structures of Li dendrites at the edge region to form "dead Li" (Supplementary Fig. 7). The thickness of deposits reaches up to $20 \mu \mathrm{m}$ with a needle-like structure (Fig. 2d). In contrast, the Li deposits on the Cu collector in the MIP electrolyte display uniform surface without sharp structure even at the edge regions (Fig. $2 \mathrm{~g}$ ), and the thickness is only $10 \mu \mathrm{m}$ with a highly dense distribution (Fig. $2 \mathrm{~h}$ ), clearly demonstrating the homogeneous Li plating is associated with the ionic self-concentrating on the montmorillonite. The element mapping displays the montmorillonite is involved in the $\mathrm{Li}$ deposition (Supplementary Fig. 8). Therefore, scanning electron microscope coupling with focused ion beam (FIB-SEM) was employed to analysis the Li deposits (Supplementary Fig. 9). It can be clearly observed that the montmorillonite can be served as heterogeneous nucleation seeds for adsorbing $\mathrm{Li}$ ions to enable a uniform deposition rather than forming large cluster or $\mathrm{Li}$ dendrites due to the low diffusion energy barrier for $\mathrm{Li}$ ion migratory.
Additionally, a $\mathrm{Li} \| \mathrm{Cu}$ cuvette-type optical cell was also fabricated to investigate the morphology of the $\mathrm{Cu}$ surface in both electrolytes during the Li deposition process through in situ optical microscopy. The images recorded at different stages are displayed in Fig. 2i, j. The $\mathrm{Li} \| \mathrm{Cu}$ cell was subjected to a high current density of $3 \mathrm{~mA} \mathrm{~cm}^{-2}$. In the beginning, the surface of $\mathrm{Cu}$ is smooth and bright in the ether-based electrolyte. However, $\mathrm{Li}$ dendrites appear immediately upon application of the current. As time goes on, numerous moss-like dendrites are formed on the $\mathrm{Cu}$ surface. In comparison, uniform electrodeposition of $\mathrm{Li}$ is obtained in the MIP-based system at high current density, leading to a flat surface without dendritic structures. This observation suggests that the MIP-based electrolyte can effectively suppress the formation of Li dendrites in a Li-metal rechargeable battery.

$\mathrm{Li}$ plating/stripping performance on $\mathrm{Cu}$ foil in MIP electrolyte. $\mathrm{Li} \| \mathrm{Cu}$ cells are fabricated to examine the cycling stability and Coulombic efficiencies for different electrolytes. Herein, the capacity of $1 \mathrm{mAh} \mathrm{cm}^{-2} \mathrm{Li}$ deposits is plated on the surface of $\mathrm{Cu}$ foil, and then stripped during the charge to $0.5 \mathrm{~V}$. When operated at $0.5 \mathrm{~mA} \mathrm{~cm}^{-2}$, the $\mathrm{Li} \| \mathrm{Cu}$ cell with the MIP electrolyte demonstrates a stable plating/stripping voltage profile and a steady Coulombic efficiency of $98 \%$ for more than 300 cycles (Fig. 3a). In comparison, the cells with ether-based electrolyte display obviously inferior Coulombic efficiencies and very fluid charge/discharge voltage profiles (Supplementary Fig. 10). Furthermore, the benefits of MIP-based electrolyte are examined 

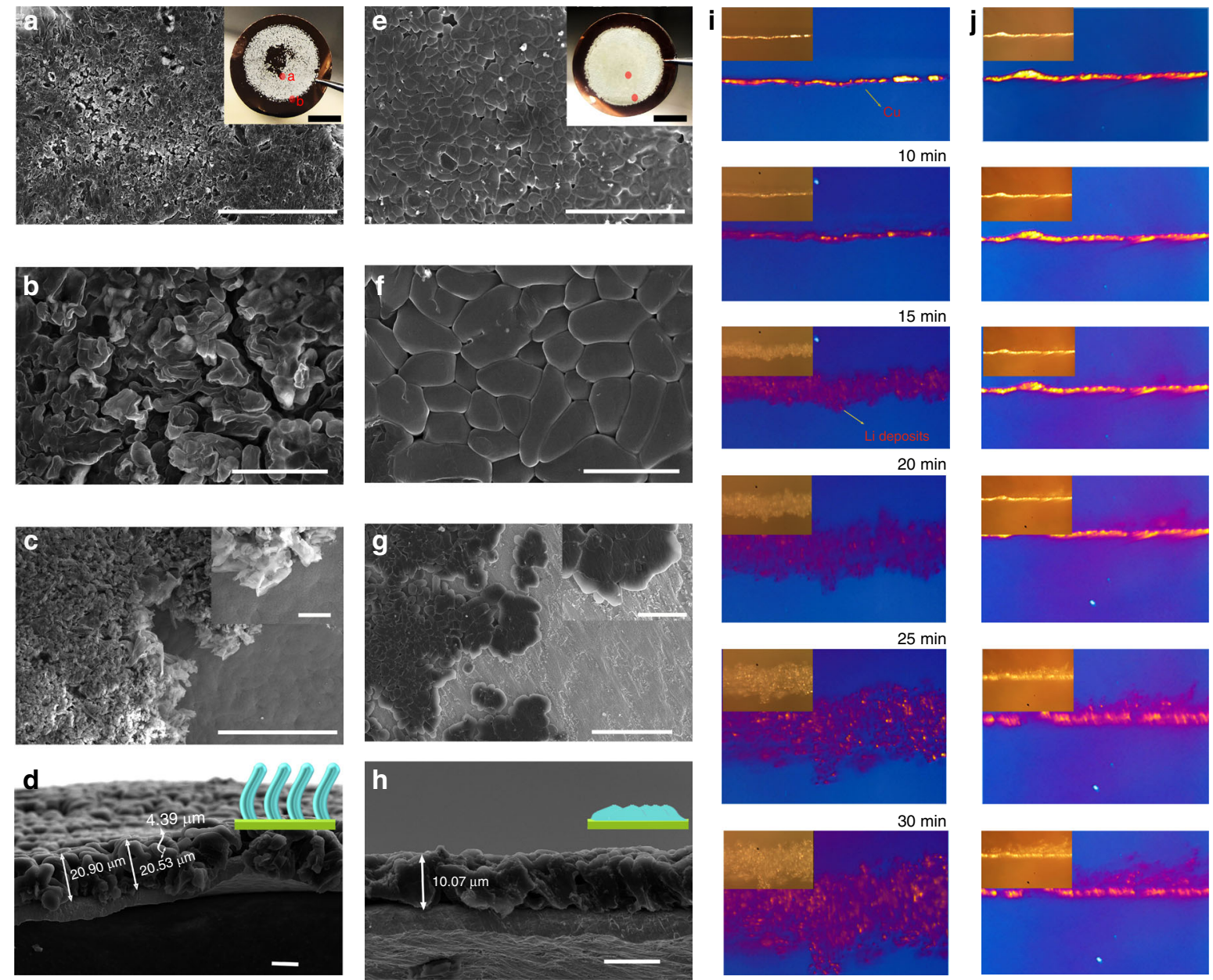

Fig. 2 The morphology of Li deposition on Cu surface. SEM images of a-d and $\mathbf{e}-\mathbf{h}$ show the Li deposits in ether-based and MIP-based electrolyte with $2 \mathrm{mAh} \mathrm{cm}-2$ at $0.5 \mathrm{~mA} \mathrm{~cm}^{-2}$, respectively. The inset digital images in $\mathbf{a}$ and $\mathbf{e}$ show the typical Li deposit distribution after plating without and with tuning $\mathrm{Li}$ ion distribution. The SEM images of $\mathbf{a}, \mathbf{b}$ and $\mathbf{e}, \mathbf{f}$ show ether-based and MIP-based in plane Li deposit region, respectively, marked red a in digital image. $\mathbf{c}, \mathbf{g}$ The edge Li deposition (marked red b) of ether-based and MIP-based electrolyte, respectively. $\mathbf{d}, \mathbf{h}$ The cross section of Li deposition in ether-based and MIP-based, respectively. The Li deposition in ether-based electrolyte shows columnar structures and the thickness is $\sim 20 \mu \mathrm{m}$; The Li deposition in MIPbased electrolyte shows the thickness $(\sim 10 \mu \mathrm{m})$ is only half of that in ether-based electrolyte, implying more dense lithium formation. the inset image in $\mathbf{d}$, h shows growth model. $\mathbf{i}, \mathbf{j}$ Optical microscope images of the Li deposits in ether (left column) and MIP (right column) -based electrolyte at 0, 10, 15, 20, 25 , and $30 \mathrm{~min}$ at a current rate of $3 \mathrm{~mA} \mathrm{~cm}^{-2}$. Scale bar, $(\mathbf{a}, \mathbf{c}, \mathbf{e}, \mathbf{g}) 50 \mu \mathrm{m},(\mathbf{b}, \mathbf{d}, \mathbf{f}, \mathbf{h}) 10 \mu \mathrm{m}$. The scale bar in the inset, (a, e) $0.5 \mathrm{~cm},(\mathbf{c}, \mathbf{g}) 10 \mu \mathrm{m}$

with harsh terms via rate performance in which the same current density $\left(0.5 \mathrm{~mA} \mathrm{~cm}^{-2}\right)$ for different capacity $\left(0.5-5 \mathrm{mAh} \mathrm{cm}^{-2}\right)$ and the same platting/stripping time $(1 \mathrm{~h})$ for different current density $\left(0.5-4 \mathrm{~mA} \mathrm{~cm}^{-2}\right)$. Clearly, the MIP-based electrolyte can maintain stable plating/stripping voltage profiles even at the high platting capacity of $5 \mathrm{mAh} \mathrm{cm}^{-2}$ (Fig. 3b) and high platting/stripping current density of $4 \mathrm{~mA} \mathrm{~cm}^{-2}$ (Fig. 3c). Subsequently, $4 \mathrm{mAh} \mathrm{cm}^{-2}$ and $2 \mathrm{mAh} \mathrm{cm}^{-2} \mathrm{Li}$ are deposited on $\mathrm{Cu}$ surface for MIP and ether-based electrolyte, respectively, and then $2 \mathrm{mAh} \mathrm{cm}^{-2}$ and $1.5 \mathrm{mAh} \mathrm{cm}^{-2} \mathrm{Li}$ deposition for MIP and ether-based electrolyte, respectively, are conducted for symmetrical tests. As shown in Fig. 3d and Supplementary Fig. 11, the cell with MIPbased electrolyte exhibits a stable voltage plateau more than 600 h. In sharp contrast, the cell using ether-based electrolyte delivers severe voltage hysteresis as the cycling time is only $250 \mathrm{~h}$ (Fig. 3d). The severely fluctuation of voltage profiles may generate from the reiterated formation/extinction of lithium dendrites, resulting in the electrical disconnection.

The average Coulombic efficiency of $\mathrm{Li} \| \mathrm{Cu}$ cell is investigated according to the way reported by Aurbach et al. ${ }^{34}$. As shown in Fig. 3e, the cell with MIP-based electrolyte delivers average Coulombic efficiency of $98.6 \%$, which is much higher than that in both the ether-based (96.1\%) and PEO-based (93.6\%) electrolyte. The higher Coulombic efficiency of the cell with MIP-based electrolyte reveals the lithium utilization can be improved during Li deposition/dissolution. Additional electrochemical impedance (EIS) spectroscopy was conducted for the Li||Cu cells after plating lithium with a capacity of $1.0 \mathrm{mAh} \mathrm{cm}^{-2}$ at a current density of $1.0 \mathrm{~mA} \mathrm{~cm}^{-2}$ (Supplementary Fig. 12). After cycling, the cells with MIP-based electrolyte exhibited a small charge transfer impedance $\left(R_{c t}\right)$, while the impedances of the cell with etherbased electrolyte fluctuated largely, thus demonstrating the importance of the stable interfacial impedance induced by the MIP-based electrolyte.

The symmetrical Li||Li cells are also employed for exploring the long-standing operation stability during Li plating/stripping. As displayed in Fig. 3g, the cell with MIP-based electrolyte holds a stable cycling for $1200 \mathrm{~h}$ at a current density of $1.0 \mathrm{~mA} \mathrm{~cm}^{-2}$, presenting a steady Li plating and striping with a slight polarization $(10 \mathrm{mV})$. However, the cells with other electrolyte exhibit severely fluctuating voltages, which can be attributed to the ever-changing and repeated growth/corrosion interfaces between Li anode and electrolytes (Supplementary Fig. 13). The large voltage hysteresis at early stage mainly originates from the 
a
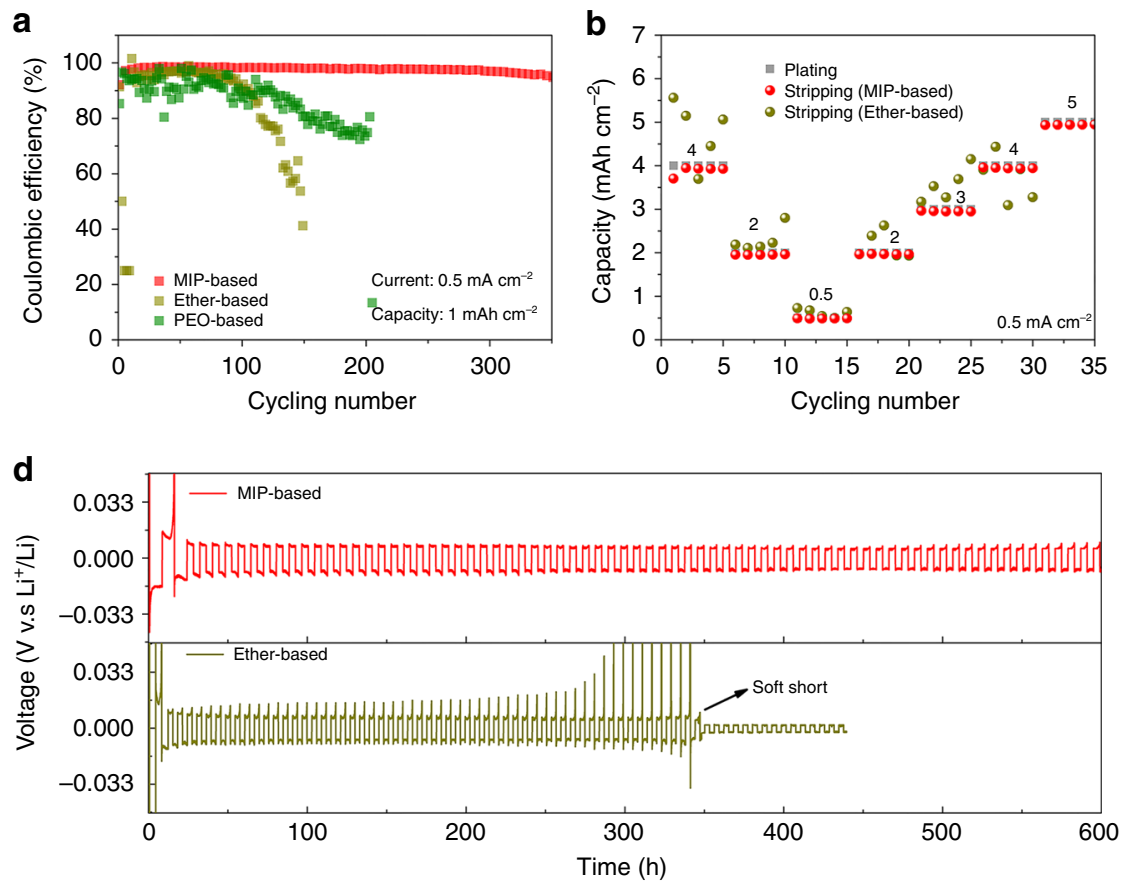

f

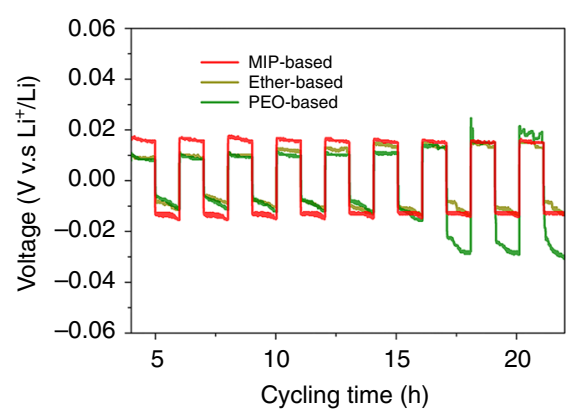

g

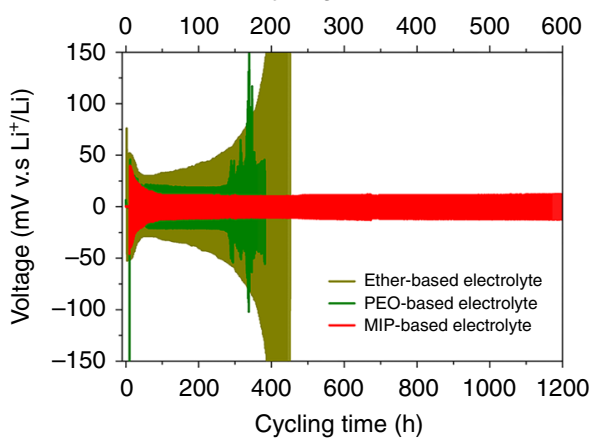

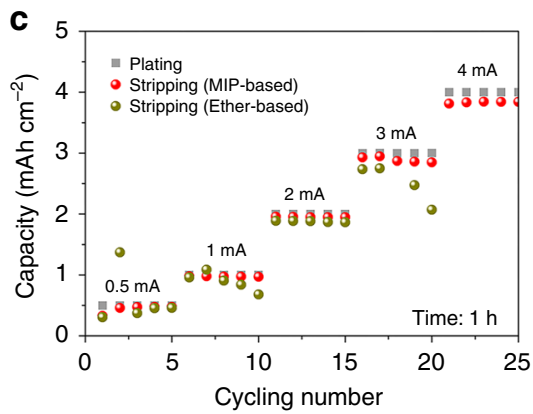

e

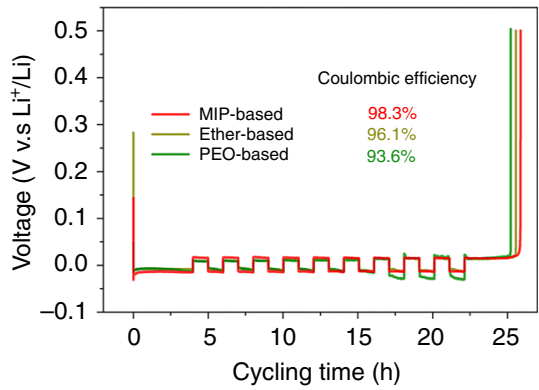

Fig. 3 Electrochemical cycling performance of Li\|Cu and Li\|Li cells. a Cycling performance of Li\|Cu with $1 \mathrm{mAh} \mathrm{cm}^{-2}$ at current density of $0.5 \mathrm{~mA} \mathrm{~cm}^{-2}$, and $\mathbf{b}$ the corresponding rate performance. $\mathbf{c}$ The rate performance with different current density at one hour. $\mathbf{d}$ The symmetrical tests of Li\|C $\mathrm{u}$ where $4 \mathrm{mAh} \mathrm{cm}^{-2}$ and $3 \mathrm{mAh} \mathrm{cm}{ }^{-2} \mathrm{Li}$ were firstly deposited on $\mathrm{Cu}$ surface for MIP and ether-based electrolyte, respectively, and then half Li deposits are conducted. e Voltage-time curves to calculate the average Coulombic efficiency of LillCu cells at $0.5 \mathrm{~mA} \mathrm{~cm}^{-2}$. $\mathbf{f}$ The enlarged view of e from $4 \sim 24 \mathrm{~h}$. $\mathbf{g}$ Charge-discharge curves of symmetrical Li||Li cells at $1 \mathrm{~mA} \mathrm{~cm}{ }^{-2}$. The morphology of Li anodes after plating in fresh (h), MIP-based (i), PEO-based (j), ether-based (k) electrolyte. Scale bar, $50 \mu \mathrm{m}$

nucleation of lithium deposition and the formation of initial SEI to passivate the lithium anode 35,36 . Figure $3 \mathrm{~h}-\mathrm{k}$ shows the morphology of Li anodes after plating in MIP-, PEO- and etherbased electrolyte, respectively. Clearly, smooth and flat morphology can be observed on both the surface and cross-sectional areas of the deposited Li in the MIP-based electrolyte (Supplementary Fig. 14). Moreover, the symmetrical Li||Li cells still deliver stable $\mathrm{Li}$ plating/stripping in the MIP-based electrolyte for $200 \mathrm{~h}$ at $1.0 \mathrm{~mA} \mathrm{~cm}^{-2}$ after resting MIP-based electrolyte for one month in glove box, confirming the stability of the montmorillonite dispersed in the electrolyte (Supplementary Fig. 15). Lithiumsulfur battery with MIP-based electrolyte demonstrates a higher average Coulombic efficiency $(99.62 \%)$ than that of the battery using ether-based electrolyte (95.63\%) during 100 cycles, further revealing the role of montmorillonite in the electrolyte to stabilize Li metal anode (Supplementary Fig. 16).

Transfer mechanism of lithium ions in MIP-based electrolyte. To better understand the $\mathrm{Li}$ ion transfer behavior, density functional theory (DFT) calculations are carried out. In Fig. 4a, the charge density distribution of $\mathrm{Li}$ ions adsorbing on the montmorillonite and $\mathrm{Li}<001>$ surface was investigated, showing that the interaction between montmorillonite and lithium ions is of ionic nature, while the bond between $\mathrm{Li}$ ion and lithium is metallic. Subsequently, the binding energies for different surfaces are compared. The binding energies of $\mathrm{Li}$ ions on montmorillonite surface, $\mathrm{Li}<001>, \mathrm{Li}<110>, \mathrm{Li}<111>$ surface and PEO are $2.66,1.37,1.35,1.67$, and $0.59 \mathrm{eV}$, respectively (Fig. 4b). The higher binding energy of Li ions on montmorillonite than that on lithium crystal plane suggests that the $\mathrm{Li}$ ions are adsorbed preferentially on the surface of montmorillonite rather than onto the Li surface during Li deposition. Once adsorption, the Li ions tend to accumulate to form large dendrites, or spread out uniformly with no dendrite generation, depending on the diffusion property of the Li ions on the adsorbents. To further investigate the Li ion diffusion behavior, the migratory barriers of $\mathrm{Li}$ ions on various surfaces are calculated and compared. The calculated results indicate the $\mathrm{Li}$ ions preferred aggregate on the $\mathrm{Li}<111>$ crystal 
a

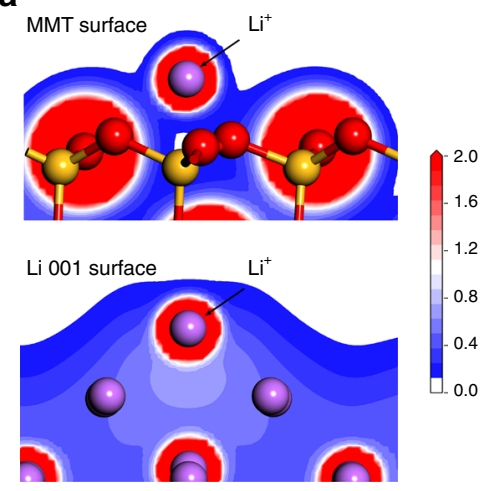

b

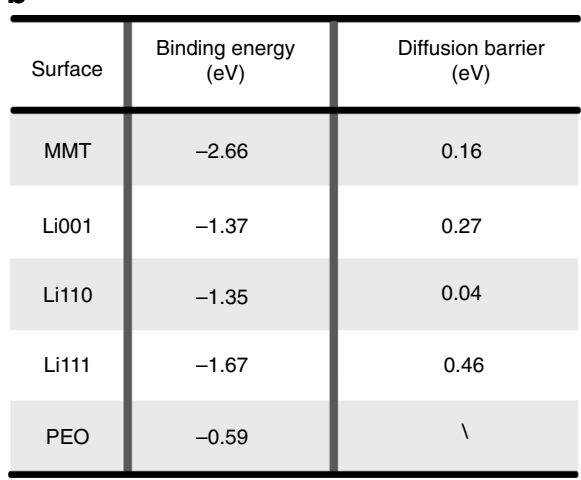

C

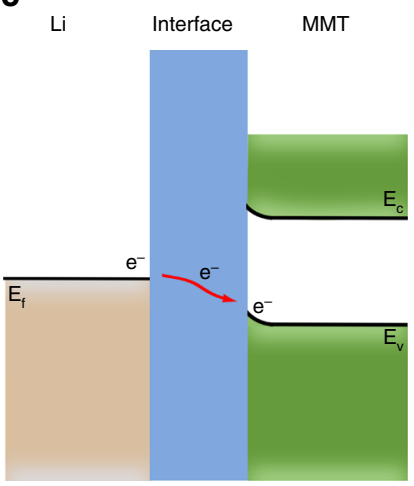

d
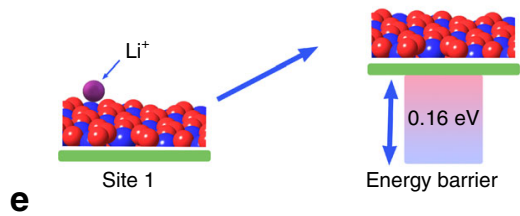

Transitional site
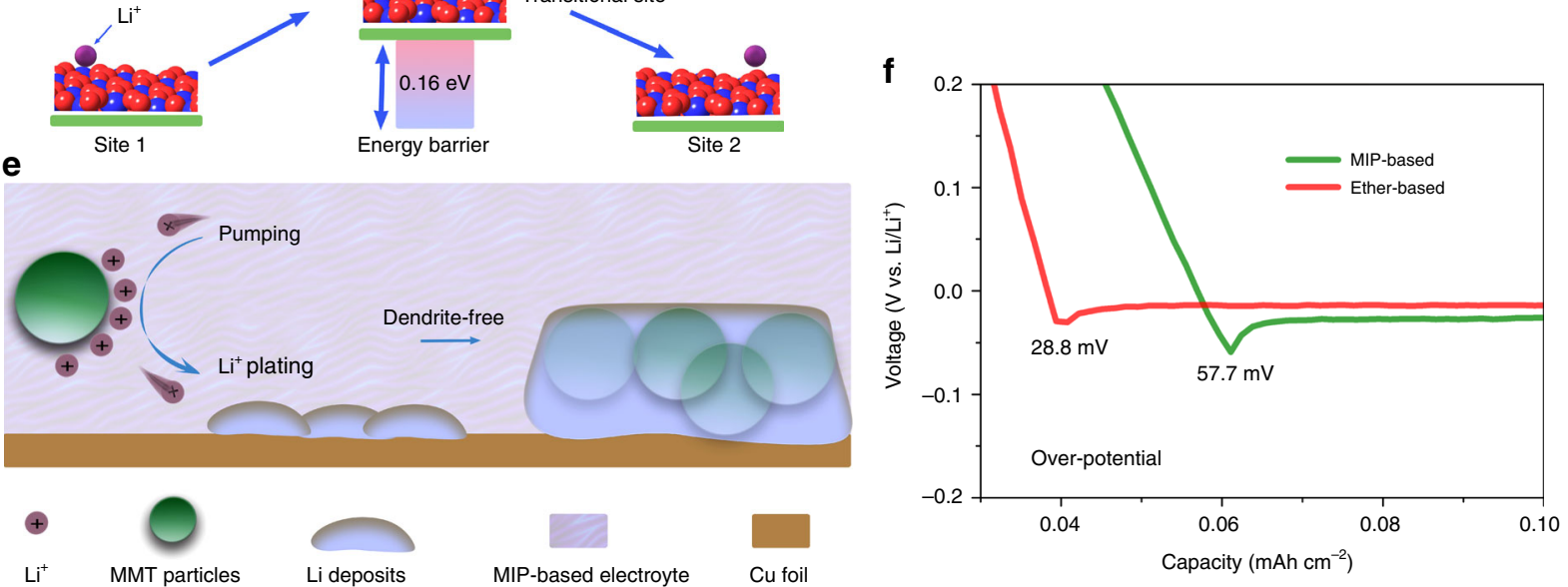

Fig. 4 Lithium ion transfer mechanism on montmorillonite surface. a The charge distribution of Li ions on montmorillonite and Li <001> surface, respectively. $\mathbf{b}$ The binding energy and diffusion barrier of $\mathrm{Li}$ ion on different material surface. c The schematic energy band diagram at the Li/ montmorillonite hetero-junction upon contact that shows electron transfer at the interface. $\mathbf{d}$, e Migrating behavior of Li ions on montmorillonite surface and bulk electrolyte, respectively. $\mathbf{f}$ The over-potential of Lil|Cu cell using MIP- and ether-based electrolyte

plane due to the highest diffusion barriers compared with $\mathrm{Li}$ $<001>, \mathrm{Li}<110>$ crystal plane (Supplementary Fig. 17), causing higher concentration at its tip, consistent with the reported value $^{37}$. In contrast, the montmorillonite owns unique layered structure with low migratory barrier $(0.16 \mathrm{eV})$, indicating that the adsorbed Li ions on surface of montmorillonite can easily diffuse and distribute uniformly (Fig. 4d). The energy band diagram demonstrates that once Li and montmorillonite contact with each other, the montmorillonite can serve as heterogeneous nucleation seeds for wetting lithium metal due to the electron transfer at the interface ${ }^{38}$, leading to dendrite-free morphology (Fig.4c). Overall, transfer mechanism of lithium ions in MIP-based electrolyte and the following deposition on the $\mathrm{Cu}$ surface can be depicted in three steps (Fig. 4e): (1) Because of the stronger affinity of montmorillonite $(2.66 \mathrm{eV})$ than both metallic $\mathrm{Li}$ and PEO, Li ions will self-concentrate near the surface of montmorillonite, in accordance with the trend in the Zeta potential $(+26 \mathrm{mV})$. (2) The adsorbed Li ions will detach, resulting in slightly elevated over-potential $(\sim 57.7 \mathrm{mV})$ as shown in Fig. 4f, then migrate from montmorillonite to the surface of the $\mathrm{Cu}$ current collector to produce Li deposits. (3) Because the montmorillonite is immobilized by PEO in the electrolyte, the formed Li deposits can contact and wet the surface of montmorillonite particles (Supplementary Figs. 7-8), and then grow along the surface, resulting in dendrite-free anodes.
Finite element simulation (FES) of Li-ion concentration profiles and current density vector (CDV). The FES is then used to study the kinetic equilibrium of Li-ion concentration and the CDV with a constant Li consumption. As shown in Fig. 5a, the lithium ion distribution within the ether-based electrolyte exhibits a typical concentration polarization region with a thickness of $\sim 5$ $\mu \mathrm{m}$ along the $\mathrm{y}$ axis, where the lithium ion concentration is significantly lower than its initial concentration $\mathrm{C}_{0}(1 \mathrm{M})$. The lower lithium ion distributions at the interface of the current collector/ electrolyte than that at other regions are more likely to result in the selective deposition of lithium ions, leading to the growth of dendrite during Li platting 9 . In contrast to the ether-based electrolyte, the concentration polarization of lithium ions within MIP-based electrolyte are significantly compressed (Fig. 5b) due to the self-concentrate of lithium ions on the surface of montmorillonite which pumps lithium ions during plating. As a result, the lithium ion concentration at the current collector/ electrolyte interface in MIP-based electrolyte is enriched causing homogeneous distribution of lithium ion at the surface of anode. Figure $5 \mathrm{c}$ shows the lithium ion concentration along the $y$ direction of the electrode surface in both the ether- and MIPbased electrode. Clearly, the lithium ion concentration is more stable in the MIP-based electrolyte than that in the ether-based electrolyte, suggesting the uniform lithium ion distribution within the MIP-based electrolyte due to its self-concentrate on the 
a Li-ion concentration

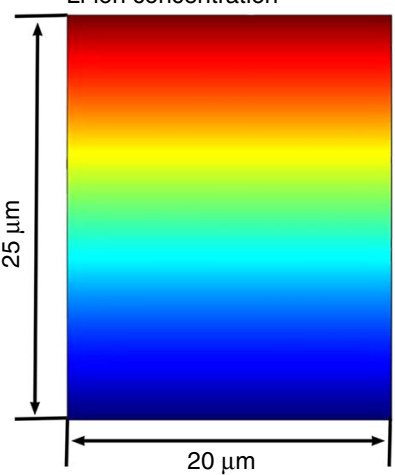

d

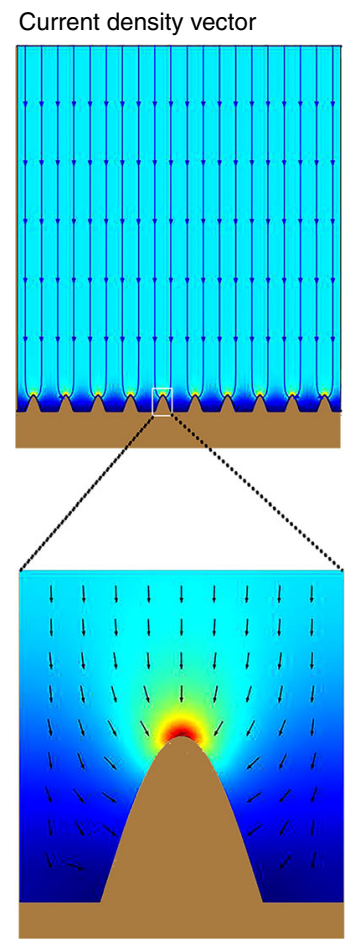

Ether-based b

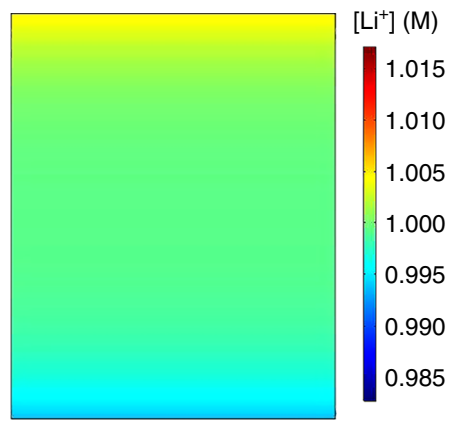

e

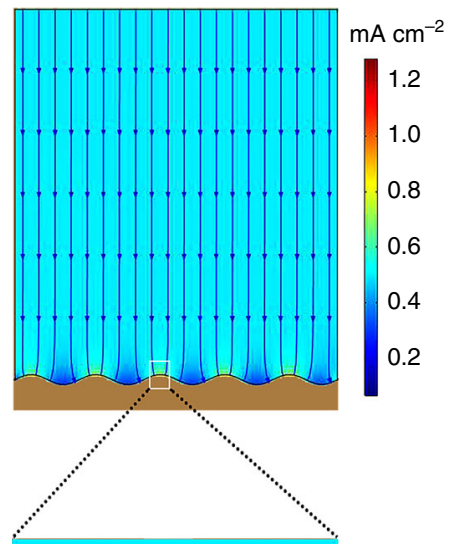

C

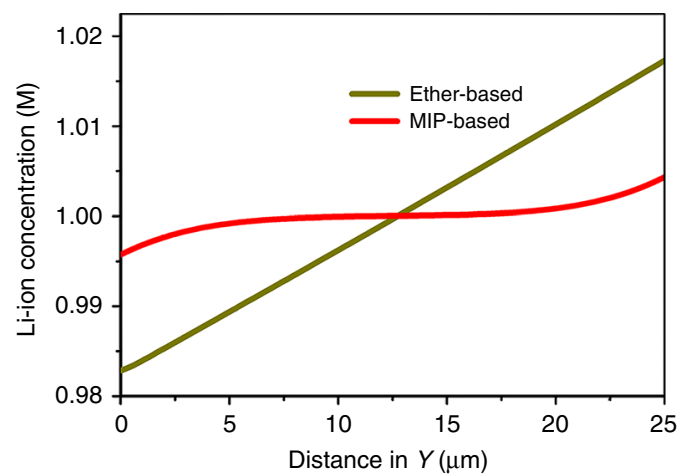

f Ether-based
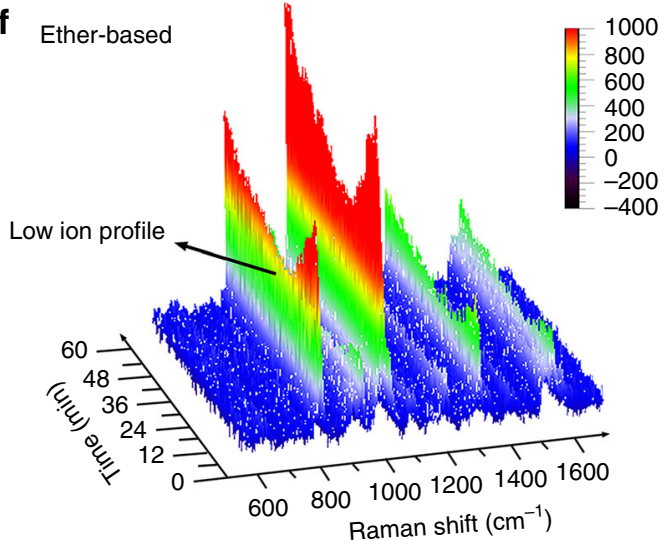

g

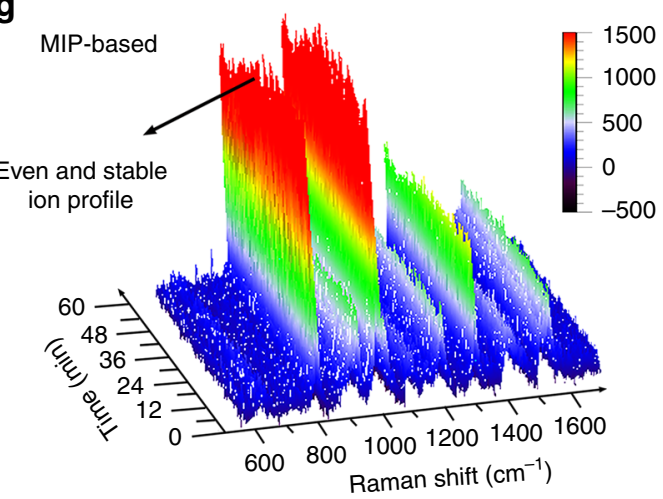

Fig. 5 Finite element simulation and in situ Raman to observe the interface information. a, b Equilibrium Li-ion concentration profiles with constant-

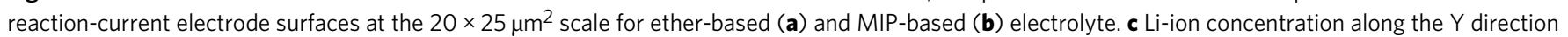
in (a, b). d, e Current density vector profiles with constant current $0.5 \mathrm{~mA} \mathrm{~cm}{ }^{-2}$ at the $20 \times 25 \mu \mathrm{m}^{2}$ half cell electrodeposition system for ether-based (d) and MIP-based (e) electrolyte. The $X$ and $Y$ axes represent the directions parallel and perpendicular to the electrode, respectively. $\mathbf{f}$, $\mathbf{g}$ In situ Raman spectra of ether and MIP-based electrolyte, respectively. The Raman band at $720-760 \mathrm{~cm}^{-1}$ is ascribed to the TFSI- band

montmorillonite surface, which will bring even Li plating and thus dendrite-free $\mathrm{Li}$ anode.

The temporal evolution of Li plating is subsequently performed via studying the CDV. The simulated structural model of $\mathrm{Li}$ deposition (Fig. 5d, e and Supplementary Fig. 18) are designed based on the experimentally observed morphology of the asdeposited Li with needle-like and flat surface (Fig. 2d, h) in etherand MIP-based electrolyte, respectively. Profiling of the Li-ion concentration (Supplementary Fig. 18) shows the Li-ions in the ether-based electrolyte tend to accumulate at the top of the $\mathrm{Li}$ protuberance (Supplementary Fig. 18a), causing unevenness of ion distribution (Supplementary Fig. 19). In contrast, in MIPbased electrolyte (Supplementary Fig. 18b), the Li-ions displays more even distribution at the interface, which benefits the $\mathrm{Li}$ depositing. By studying the CDV line, it can be found that the current density $\left(>1.2 \mathrm{~mA} \mathrm{~cm}^{-2}\right)$ at the top of the Li protuberance in the ether-based electrolyte (Fig. 5d) far exceeds the simulated constant current density $\left(0.5 \mathrm{~mA} \mathrm{~cm}^{-2}\right)$, and such a high current density will further aggravate the growth of lithium dendrites. Moreover, even at a high current density of $3 \mathrm{~mA} \mathrm{~cm}^{-2}$ (Supplementary Fig. 20), the MIP-based electrolyte still delivers more uniform surface current distribution, enabling a dendritefree Li anode.

In situ Raman observation of the electrochemical interface during plating process. The visualization of even $\mathrm{Li}$ ion profile distribution in MIP-based electrolyte at the $\mathrm{Cu}$ negative interface is 
further demonstrated via in situ Raman spectroscopy measurement in which the Raman band at $741.8 \mathrm{~cm}^{-1}$ is ascribed to the coupled $\mathrm{CF}_{3}$ bending and $\mathrm{S}-\mathrm{N}$ stretching in $\mathrm{TFSI}^{-}$(Supplementary Fig. 21) ${ }^{39}$. For ether-based electrolyte, we observe a significant change of the spectrum intensity with increasing Li plating capacity. At the early stage of plating, the Raman beam mainly focused on the neighborhood of $\mathrm{Cu}$ surface (Supplementary Fig. 22a), and the detected signal is ascribed to the bulk electrolyte, resulting in considerable intensity of Raman signal. The signal intensity, however, dramatically decreases with the growth of Li deposits. Especially, the intensity reduces to half of the initial value after plating for $30 \mathrm{~min}$ because the interface gradually moves close to the focused point of Raman beam (Supplementary Fig. 22a). The decreased signal intensity at this time presents the real ion distribution at the interface, ${ }^{29}$ providing strong evidence for uneven ion distribution on the anode surface compared with the bulk electrolyte (Supplementary Fig. 22b). In contrast, benefiting from the pumping feature of montmorillonite for self-concentrate of $\mathrm{Li}$ ions, ion distribution at the electrochemical interface is not affected, which is confirmed by the nearly constant intensity of Raman signal (Supplementary Fig. 23). The increased ion concentration (Supplementary Fig. 24) favors further delay of the Sand's time and provides stable and even plating.

\section{Discussion}

In summary, we proposed a promising strategy to tune ion distribution for $\mathrm{Li}$ dendrite-free deposition by introducing montmorillonite into the bulk electrolyte as nucleophilic medium. The redistribution of $\mathrm{Li}$ ion effectively suppresses the formation of ion barren region on the surface of anode and further delays Sand's time by increasing the ion concentration at the electrode/electrolyte interface. This mechanism is demonstrated by FES and in situ Raman measurement which shows that the intensity at the deposition/electrolyte interface in ether-based electrolyte is significantly lower than that of the bulk electrolyte at the high current density of $3 \mathrm{~mA} \mathrm{~cm}^{-2}$. In contrast, for MIP-based electrolyte, flat and stable Raman spectra can be observed during Li plating, which means the barren area has been turned into fertile fields by the MIP-based electrolyte. The fertile ion distribution can offset the negative effects induced by inhomogeneity on the surface of the current collector. As the deposition continues, the montmorillonite serves as the heterogeneous nucleation seeds for adsorbing $\mathrm{Li}$ ions, leading to a uniform deposition rather than large cluster or $\mathrm{Li}$ dendrites. The stable $\mathrm{Li}$ platting/stripping renders an enhanced electrochemical performance even at high platting/stripping capacity of $5 \mathrm{mAh} \mathrm{cm}^{-2}$, and the $\mathrm{Li} \| \mathrm{Cu}$ cell can also deliver a high Coulombic efficiency. When employing Aurbach's methods for probing the Coulombic efficiency ${ }^{34}$, the average Coulombic efficiency of the cell with MIP-based electrolyte can reach up to $98.3 \%$, which is much higher than that of the cell using ether-based electrolyte (96.1\%). This study suggests that the distribution of $\mathrm{Li}$ ion at the deposition/electrolyte interface is a decisive factor for $\mathrm{Li}$ ion plating. Guiding even $\mathrm{Li}$ ion distribution in bulk electrolyte by nucleophilic inorganicinterlamellar montmorillonite material offers a promising route to suppress dendrite growth and enable ultra-stable Li platting/ stripping.

\section{Methods}

MIP-based electrolyte preparation. In an Ar-filled glove box, $3.3 \mu \mathrm{M}$ PEO (molecular weight: $300,000 \mathrm{~g} \mathrm{~mol}^{-1}$, Aladdin) was dissolved in $10 \mathrm{~mL}$ ether-based electrolyte for which 1.0 M lithium bis(trifluoromethylsulfonyl)imid (LiTFSI) was dissolved in DOL/DME (1:1 by volume; $\mathrm{DOL}=1,3$-dioxalane; DME = dimethylether) with $1 \mathrm{wt} \% \mathrm{LiNO}_{3}$, and then $100 \mathrm{mg}$ KFS-type montmorillonite particles (Aladdin) were added. The obtained colloidal solution was subsequently stirred for $24 \mathrm{~h}$ at $60^{\circ} \mathrm{C}$ to ensure uniform dispersal. After that, the milk white solution, denoted as montmorillonite implant in PEO (MIP)-based electrolyte, was obtained and left in glove box for following electroplating processes and electrochemical long-term cycling tests.

Materials characterization. The structure of montmorillonite was characterized by powder X-ray diffraction (XRD) at room temperature using a UltimaIV diffractometer with CuKal radiation $(\lambda=1.4506 \AA)$ and a position-sensitive detector. Zeta potential was used to measure the shear plane's potential using NanoBrook 90Plus PALS Particle Size Analyzer (USA). The morphology of Li deposits was observed using a SEM (FEI, NANOSEI 450), operated at $3.0 \mathrm{kV}$ with the SEM samples were prepared in the glove box.

Calculations methods. The interaction among $\mathrm{PEO}$, montmorillonite, $\mathrm{Li}^{+}, \mathrm{DOL}$ and DME are calculated by the DFT implemented by DMOL3 software. Double numerical plus polarization (DNP) basis with DFT Semi-core Pseudopots treatment and a GGA-PBE function were employed in the self-consistent field calculation with a SFC tolerance of $1 \times 10^{-6}$. A short polymer chain of $\mathrm{C}_{8} \mathrm{H}_{2} \mathrm{O}_{4}$ was used to stand for PEO polymer.

Finite element simulation. The COMSOL Multiphysics software, coupling with lithium battery module simulation, was employed for the FES. The plating morphological evolution was conducted in $20 \times 25 \mu \mathrm{m}$. The electrolyte conductivity is $10^{-2} \mathrm{~S} \mathrm{~m}^{-1}$, the diffusion coefficient is $1.5 \times 10^{-10} \mathrm{~m} \mathrm{~s}^{-2}$, the lithium ion concentration is $1 \mathrm{M}$, and the surface current densities of 0.5 and $3 \mathrm{~mA} \mathrm{~cm}^{-2}$ are simulated. For the case of modification, a porous adsorption medium is added to the electrolyte, and the adsorb ability $(2.66 \mathrm{eV})$ of montmorillonite for lithium ions is based on the DFT calculations.

In situ Raman measurement. Lil|Cu cell with a quartz window was used for in situ micro-Raman spectroscopy analysis. The confocal micro-Raman spectrometer was equipped with a $785 \mathrm{~nm}$ laser source. Each spectrum was the average of two accumulations at a resolution of approximately $4 \mathrm{~cm}^{-1}$. The Raman beam is foucsed on the neiborhood of $\mathrm{Cu}$ cross section. To ensure enough $\mathrm{Li}$ plating on $\mathrm{Cu}$ surface, $3 \mathrm{mAh} \mathrm{cm}^{-2} \mathrm{Li}$ was plating at a current density of $3 \mathrm{~mA} \mathrm{~cm}^{-2}$. All spectral analyses were carried out using build-in commercial software of micro-Raman spectrometer. All Raman spectra are shown as measured without background correction.

Electrochemical cycling tests. The Li || $\mathrm{Cu}$ cells and symmetrical Li $\mid \mathrm{Li}$ cells were assembled in the argon filled glove box. The coin cells (CR-2032) were used to test the electrochemical performance. The used separator is polypropylene membranes (Celgard 2400). The coin cells were monitored using a battery cycler on CT2001A cell test instrument (Wuhan LAND Electronic Co., Ltd). And the $\mathrm{Z}-\mathrm{K}$ study was performed using CHI660E (Shanghai Chenhua instrument Co., Ltd) electrochemical workstation. The average Coulombic efficiency of Li platting/stripping was calculated based on Aurbach et al..$^{34}$. Briefly, a capacity of $2 \mathrm{mAh} \mathrm{cm}^{-2} \mathrm{Li}$ deposition was first platted on the surface of $\mathrm{Cu}$ electrode. Then, the $\mathrm{Li}$ deposition with $0.5 \mathrm{mAh} \mathrm{cm}^{-2}$ was stripped and re-deposited at a current density of $0.5 \mathrm{~mA} \mathrm{~cm}^{-2}$ for 10 cycles. The cell was stopped till the voltage exceeded $0.5 \mathrm{~V}$ vs. $\mathrm{Li} / \mathrm{Li}^{+}$as the final stripping process. The following equation is employed for calculating the average cycling efficiency.

$$
X=\left[1-\frac{X\left(Q_{1}-Q_{r}\right)}{Q_{c} N}\right] \times 100
$$

where $X$ presents the cycling efficiency (\%), $N$ is the number of cycles, $Q_{1}$ is the initial $\mathrm{Li}$ deposition (initial loading), $Q_{r}$ is the finally residual of $\mathrm{Li}$ deposition (final charging), $Q_{c}$ is the charge for the single amount of Li deposition (half cycle). For lithium sulfur battery tests, the preparation of sulfur electrode is followed our previously reported method. ${ }^{40}$ Typically, the sulfur content in S/C composite is $60 \%$ and the slurry of S/C and Poly(vinylidene fluoride) binder in a mass ratio of 9:1 in N-methyl pyrrolidone solvent was casted on the surface of the carbon coated aluminum foil. The use of electrolyte is MIP- or ether-based electrolyte.

\section{Data availability}

The authors declare that all the data supporting the findings of this study are available within the article and its Supplementary Information or from the corresponding author upon reasonable request.

Received: 7 July 2019; Accepted: 11 October 2019; Published online: 31 October 2019

\section{References}

1. Zhao, Q., Liu, X., Stalin, S., Khan, K. \& Archer, L. A. Solid-state polymer electrolytes with in-built fast interfacial transport for secondary lithium batteries. Nat. Energy 4, 365-373 (2019). 
2. Li, G. et al. Stable metal battery anodes enabled by polyethylenimine sponge hosts by way of electrokinetic effects. Nat. Energy 3, 1076-1083 (2018).

3. Liu, J. et al. Pathways for practical high-energy long-cycling lithium metal batteries. Nat. Energy 4, 180-186 (2019).

4. Yu, X. \& Manthiram, A. Enhanced interfacial stability of hybrid-electrolyte lithium-sulfur batteries with a layer of multifunctional polymer with intrinsic nanoporosity. Adv. Funct. Mater. 29, 1805996 (2018).

5. Urbonaite, S., Poux, T. \& Novák, P. Progress towards commercially viable Li-S battery cells. Adv. Energy Mater. 5, 1500118 (2015).

6. Ma, J. L. et al. Prevention of dendrite growth and volume expansion to give high-performance aprotic bimetallic Li-Na alloy- $\mathrm{O}_{2}$ batteries. Nat. Chem. 11, 64-70 (2019)

7. Liang, Y. et al. A review of rechargeable batteries for portable electronic devices. InfoMat 1, 6-32 (2019).

8. Fan, X., Liu, X., Hu, W., Zhong, C. \& Lu, J. Advances in the development of power supplies for the internet of everything. InfoMat 1, 130-139 (2019).

9. Brissot, C., Rosso, M., Chazalviel, J. N. \& Lascaud, S. Dendritic growth mechanisms in lithium/polymer cells. J. Power Sources 81, 925-929 (1999).

10. Guan, X. et al. Controlling nucleation in lithium metal anodes. Small 14, 1801423 (2018).

11. Assat, G. \& Tarascon, J. M. Fundamental understanding and practical challenges of anionic redox activity in Li-ion batteries. Nat. Energy 3, 373-386 (2018).

12. Lu, Y., Tu, Z. \& Archer, L. A. Stable lithium electrodeposition in liquid and nanoporous solid electrolytes. Nat. Mater. 13, 961-969 (2014).

13. Cheng, X. B. et al. Dendrite-free lithium deposition induced by uniformly distributed lithium ions for efficient lithium metal batteries. Adv. Mater. 28, 2888-2895 (2016).

14. Finegan, D. P. et al. In-operando high-speed tomography of lithium-ion batteries during thermal runaway. Nat. Commun. 6, 6924 (2015).

15. Kim, H. et al. Metallic anodes for next generation secondary batteries. Chem. Soc. Rev. 42, 9011-9034 (2013).

16. Guo, Y., Li, H. \& Zhai, T. Reviving lithium-metal anodes for next-generation high-energy batteries. Adv. Mater. 29, 1700007 (2017).

17. Zeng, Z. et al. Visualization of electrode-electrolyte interfaces in $\mathrm{LiPF}_{6} / \mathrm{EC} /$ DEC electrolyte for lithium ion batteries via in situ TEM. Nano Lett. 14, 1745-1750 (2014).

18. Zhou, J. et al. High-safety all-solid-state lithium-metal battery with high-ionicconductivity thermoresponsive solid polymer electrolyte. Nano Lett. 19, 3066-3073 (2019).

19. Wang, S. H. et al. Stable Li metal anodes via regulating lithium plating/ stripping in vertically aligned microchannels. Adv. Mater. 29, 1703729 (2017).

20. Li, Q., Zhu, S. \& Lu, Y. 3D porous cu current collector/Li-metal composite anode for stable lithium-metal batteries. Adv. Funct. Mater. 27, 1606422 (2017).

21. Ye, H. et al. Stable Li plating/stripping electrochemistry realized by a hybrid Li reservoir in spherical carbon granules with 3D conducting skeletons. J. Am. Chem. Soc. 139, 5916-5922 (2017).

22. Chen, W. et al. Designing safe electrolyte systems for a high-stability lithiumsulfur battery. Adv. Energy Mater. 8, 1702348 (2018).

23. Zhang, Q. et al. An alternative to lithium metal anodes: non-dendritic and highly reversible sodium metal anodes for Li-Na hybrid batteries. Angew. Chem. Int. Ed. 57, 14796-14800 (2018).

24. Ding, F. et al. Dendrite-free lithium deposition via self-healing electrostatic shield mechanism. J. Am. Chem. Soc. 135, 4450-4456 (2013).

25. Zhu, B. et al. Poly(dimethylsiloxane) thin film as a stable interfacial layer for high-performance lithium-metal battery znodes. Adv. Mater. 29, 1603755 (2017).

26. Duan, J. et al. Lithium-graphite paste: an interface compatible anode for solidstate batteries. Adv. Mater. 31, e1807243 (2019).

27. Zhang, H. et al. Lithiophilic-lithiophobic gradient interfacial layer for a highly stable lithium metal anode. Nat. Commun. 9, 3729 (2018).

28. Zhang, W. et al. Tuning the LUMO energy of an organic interphase to stabilize lithium metal batteries. ACS Energy Lett. 4, 644-650 (2019).

29. Choi, N. S. et al. Challenges facing lithium batteries and electrical double-layer capacitors. Angew. Chem. Int. Ed. 51, 9994-10024 (2012).

30. Li, G. et al. Electrokinetic phenomena enhanced lithium-ion transport in leaky film for stable lithium metal anodes. Adv. Energy Mater. 9, 1900704 (2019).

31. Moreno-Sader, K., García-Padilla, A., Realpe, A., Acevedo-Morantes, M. \& Soares, J. B. P. Removal of heavy metal water pollutants $\left(\mathrm{Co}^{2+}\right.$ and $\left.\mathrm{Ni}^{2+}\right)$ using polyacrylamide/sodium montmorillonite (PAM/Na-MMT) nanocomposites. ACS Omega 4, 10834-10844 (2019).
32. Atta, A. M., Al-Lohedan, H. A., Alothman, Z. A., Abdel-Khalek, A. A. \& Tawfeek, A. M. Characterization of reactive amphiphilic montmorillonite nanogels and its application for removal of toxic cationic dye and heavy metals water pollutants. J. Ind. Eng. Chem. 31, 374-384 (2015).

33. Lei, T. et al. Inhibiting polysulfide shuttling with a graphene composite separator for highly robust lithium-sulfur batteries. Joule 2, 2091-2104 (2018).

34. Aurbach, D. The correlation between surface chemistry, surface morphology, and cycling efficiency of lithium electrodes in a few polar aprotic systems. J. Electrochem. Soc. 136, 3198 (1989).

35. Pei, A., Zheng, G., Shi, F., Li, Y. \& Cui, Y. Nanoscale nucleation and growth of electrodeposited lithium metal. Nano Lett. 17, 1132-1139 (2017).

36. Miao, R. et al. Novel dual-salts electrolyte solution for dendrite-free lithiummetal based rechargeable batteries with high cycle reversibility. J. Power Sources 271, 291-297 (2014).

37. $\mathrm{Li}, \mathrm{Y}$. et al. Atomic structure of sensitive battery materials and interfaces revealed by cryo-electron microscopy. Science 358, 506-510 (2017).

38. Park, K. \& Goodenough, J. B. Dendrite-suppressed lithium plating from a liquid electrolyte via wetting of $\mathrm{Li}_{3} \mathrm{~N}$. Adv. Energy Mater. 7, 1700732 (2017).

39. Pang, Q. et al. Tuning the electrolyte network structure to invoke quasi-solid state sulfur conversion and suppress lithium dendrite formation in Li-S batteries. Nat. Energy 3, 783-791 (2018).

40. Chen, W. et al. A new type of multifunctional polar binder: toward practical application of high energy lithium sulfur batteries. Adv. Mater. 29, 1605160 (2017).

\section{Acknowledgements}

We gratefully acknowledge the support from the National Natural Science Foundation of China (51722204), National Key Basic Research Program of China (2014CB931702).

\section{Author contributions}

J.X., C.Y., and M.L. conceived and designed this work. W.C., Y.H. and X.W. synthesized the MIP-based electrolyte and wrote the paper. W.L. performed the first-principle calculations. T.L., Z.L., and M.Z. measured the electrochemical cycling performance. J.H., X.D., Y.Y. and W.H. constructed the in situ Raman spectra. W.C., Y.H., C.L. and W.Z conducted SEM analysis. All authors participated in analysis of the experimental data and discussions of the results, as well as editing the manuscript. W.C., Y.H. and W.L. contributed equally to this work.

\section{Competing interests}

The authors declare no competing interests.

\section{Additional information}

Supplementary information is available for this paper at https://doi.org/10.1038/s41467 019-12952-6.

Correspondence and requests for materials should be addressed to M.L., J.X. or C.Y.

Peer review information Nature Communications thanks the anonymous reviewer(s) for their contribution to the peer review of this work.

Reprints and permission information is available at http://www.nature.com/reprints

Publisher's note Springer Nature remains neutral with regard to jurisdictional claims in published maps and institutional affiliations.

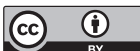

Open Access This article is licensed under a Creative Commons Attribution 4.0 International License, which permits use, sharing, adaptation, distribution and reproduction in any medium or format, as long as you give appropriate credit to the original author(s) and the source, provide a link to the Creative Commons license, and indicate if changes were made. The images or other third party material in this article are included in the article's Creative Commons license, unless indicated otherwise in a credit line to the material. If material is not included in the article's Creative Commons license and your intended use is not permitted by statutory regulation or exceeds the permitted use, you will need to obtain permission directly from the copyright holder. To view a copy of this license, visit http://creativecommons.org/ licenses/by/4.0/

(C) The Author(s) 2019 